\title{
Hybrid boron-10 gaseous detector for slow and fast neutron simultaneous detection
}

\author{
Stanislav Potashev ${ }^{1, *}$, Aleksandr Drachev ${ }^{2}$, Yury Burmistrov ${ }^{1}$, Sergey Karaevsky ${ }^{1}$, Aleksandr Kasparov ${ }^{1}$, Vasily \\ Ponomarev $^{1}$, and Gennady Solodukhov ${ }^{1}$ \\ ${ }^{1}$ Institute for Nuclear Research, Russian Academy of Sciences, 117312 Moscow, Russia \\ ${ }^{2}$ State Research Institute for Chemistry and Technology of Organoelement Compounds, 119991 Moscow, Russia
}

\begin{abstract}
A hybrid position-sensitive neutron detector with active $\mathrm{e}^{10} \mathrm{~B}$-layer and proportional chamber filled by the gas mixture $\mathrm{Ar}+25 \% \mathrm{CO}_{2}$ is being studied. The detector is used on the neutron source based at the linear accelerator. Recording two pairs of pulse heights which are produced in two gas gaps by ${ }^{7} \mathrm{Li}$ and ${ }^{4} \mathrm{He}$ nuclei between the anode and two cathodes gives a possibility to determine the $\mathrm{X}$ and $\mathrm{Y}$ neutron coordinates. This detector permits time-of-flight measurements. A significant difference in pulse height spectra for slow and fast (several $\mathrm{MeV}$ ) neutrons was observed in experiment. In recent experiments with ${ }^{10} \mathrm{~B}$-detector we found that (1) the pulse heights depend on the neutron energy and (2) the position of the maximum in the pulse height spectrum varies monotonically versus the maximum neutron energy.
\end{abstract}

\section{Introduction}

There are many applications of neutron positionsensitive detectors in physics [1], technology [2] and biology [3]. The choice of a kind of detector depends on the energy range of neutrons. To investigate the atomic structure in superconductors thermal neutron diffraction can be used [4]. Small angle scattering is used for the investigation of alloys [5], magnetic structures [6], protein molecules in biology [7].

Usually, ${ }^{3} \mathrm{He}$ - and ${ }^{10} \mathrm{BF}_{3}$-tube counters and their assemblies are widely used in a diffraction setup and in small angle scattering. The position sensitive proportional chamber filled by ${ }^{3} \mathrm{He}$ under high pressure [8] or ${ }^{10} \mathrm{BF}_{3}$ is more rare and expensive device. A proportional chamber and a assembly of tube counters filled by the ${ }^{3} \mathrm{He}$ gas have a high efficiency and low sensitivity to $\gamma$-rays. But they operate under a high pressure and have a thick entrance window and may experience leakage of expensive gas.

Also, scintillation detectors with the embedded ${ }^{6} \mathrm{Li}$ are developed [9-11]. They are used to detect slow neutrons. Detection of fast neutrons is important in radiation protection and in many branches of physics. As a rule, a scintillation detector of a recoil proton is used for the detection of fast neutrons [12]. However, it has a high sensitivity of the scintillation detector to $\gamma$-rays and $\mathrm{x}$-rays which makes it difficult to detect neutrons. Besides that, the substance of scintillation detector distorts the neutron distribution. The pulse height is not proportional to an ionization loss.
Recently, novel hybrid detectors for slow neutrons and for neutron flux measurements have been developed $[13,14]$. They have sensitivity only to neutrons. Therefore, they are suited to study rare nuclear reactions. The detector is based on the ${ }^{10} \mathrm{~B}$ solid layers combined with a proportional ion chamber $[15,16]$. The neutron cross section on the ${ }^{10} \mathrm{~B}$ nucleus has a very important feature, namely, that it sharply increases between 1 and $7 \mathrm{MeV}$ up to $\sim 1 \mathrm{~b}$. Therefore, the ${ }^{10} \mathrm{~B}$-detector, unlike the ${ }^{3} \mathrm{He}$-detector, can also be used as a fast neutron detector [17].

In Section 2 we discuss the concept and design of our detector. In Section 3 we discuss recent experiments with slow and fast neutrons. We conclude with perspectives of hybrid ${ }^{10} \mathrm{~B}$-detectors.

\section{Position-sensitive ${ }^{10} \mathrm{~B}$-detector}

The detector principle is as follows: ${ }^{10} \mathrm{~B}$ nucleus captures a neutron and forms the nucleus ${ }^{11} \mathrm{~B}^{*}$ in the excited state, which decays in two ways as shown below $\mathrm{n}+{ }^{10} \mathrm{~B} \rightarrow{ }^{11} \mathrm{~B}^{*} \rightarrow{ }^{4} \mathrm{He}(1470 \mathrm{keV})+{ }^{7} \mathrm{Li}(841 \mathrm{keV})+\gamma(476 \mathrm{keV})$ branch ratio is 0.936 for $0.025 \mathrm{eV}$; 0.3 for $1.8 \mathrm{MeV}$; 0.5 for $2.5 \mathrm{MeV}$

$\mathrm{n}+{ }^{10} \mathrm{~B} \rightarrow{ }^{11} \mathrm{~B}^{*} \rightarrow{ }^{4} \mathrm{He}(1770 \mathrm{keV})+{ }^{7} \mathrm{Li}(1013 \mathrm{keV})$

branch ratio is 0.064 for $0.025 \mathrm{eV}$; 0.7 for $1.8 \mathrm{MeV}$; 0.5 for $2.5 \mathrm{MeV}$.

\footnotetext{
* Corresponding author: potashev@,inr.ru
} 
The $3-\mu \mathrm{m}{ }^{10} \mathrm{~B}$ layer was deposited on an inner surface of a 1-mm glass plate. The 0.2- $\mu \mathrm{m}$ Al layer was deposited on the boron layer and it forms the first cathode of the detector. A thin layer of a semiconducting polymer was deposited for the cathode protection. The concept and design of detector is demonstrated in Fig.1. Only one nucleus ${ }^{4} \mathrm{He}$ or ${ }^{7} \mathrm{Li}$ produced by the thermal neutron can penetrate to the active gas volume overcoming the remaining layers of boron and aluminum. One nucleus or both nuclei can enter the sensitive gas volume if a neutron has energy of several $\mathrm{MeV}$. The space between the first cathode and the anode of $20-\mu \mathrm{m}$ tungsten rhenium wires coated by gold serves as the first sensitive gap. The spacing between wires is equal to $2 \mathrm{~mm}$. The second sensitive gap is the space between the anode and second cathode. Both gaps are equal to $2 \mathrm{~mm}$. The neutron event occurs only if at least one nucleus gives ionization signal in the second sensitive gap.

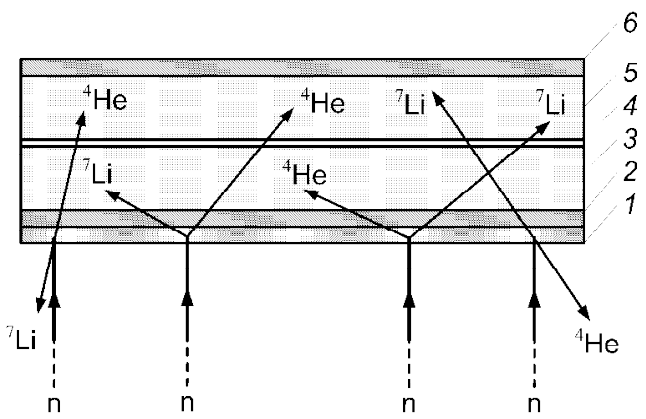

Fig. 1. ${ }^{10} \mathrm{~B} 2 \mathrm{D}$ detector concept. $1-{ }^{10} \mathrm{~B}$-layer, $3 \mu \mathrm{m} ; 2-\mathrm{Al}-$ layer, $0.2 \mu \mathrm{m} ; 3$ - 1-st sensitive gas gap of $2 \mathrm{~mm}$ thickness; 4 WRe-wires, $20 \mu \mathrm{m}$; 5 -2-nd sensitive gap of $2 \mathrm{~mm}$ thickness; 6-2 $\mathrm{mm}$ thickness fiberglass.

Various gas mixtures can be used to fill the detector. The cheap gas mixture $\mathrm{Ar}+25 \% \mathrm{CO}_{2}$ was used in experiments. It provides a long-time operation of the detector without aging. The charge division method is used to determine the $\mathrm{X}$ - and $\mathrm{Y}$-coordinates of the neutron [18]. For this aim, anode wires are connected to a circuit of distributed resistors. Partial pulse heights $\mathrm{X}_{1}$ and $\mathrm{X}_{2}$ from two ends of the circuit are used to determine the X-coordinate. Analogously, the pads of the second cathode are connected to a circuit of distributed resistors. Partial pulse heights $Y_{1}$ and $Y_{2}$ from two ends of another circuit of distributed resistors are used to determine the $\mathrm{Y}$-coordinate. A pulse height resolution of $15 \%$ and a spatial resolution has been obtained at $700 \mathrm{~V}$. The detector operates in a proportional mode at low gas gain. This provides small fluctuations in an ionization loss and, consequently, a high resolution in pulse height [19].

To determine a detector spatial resolution a cadmium mask with three $1 \mathrm{~mm}$-slits was used. Slits were in the distances separated by $20 \mathrm{~mm}$ from each other. The moderator of the neutron source was configured to the flux enriched by thermal neutrons. The ${ }^{10} \mathrm{~B}$ detector was located at a $4 \mathrm{~m}$ distance from the source centre. The ${ }^{3} \mathrm{He}$ detector served as a flux monitor. The coordinate distribution on $\mathrm{X}$-axis and $\mathrm{Y}$-axis is shown in Fig.2. The spatial resolution is $\sim 2 \mathrm{~mm}$ along $\mathrm{X}$-axis, and $\sim 4 \mathrm{~mm}$ along $\mathrm{Y}$-axis.
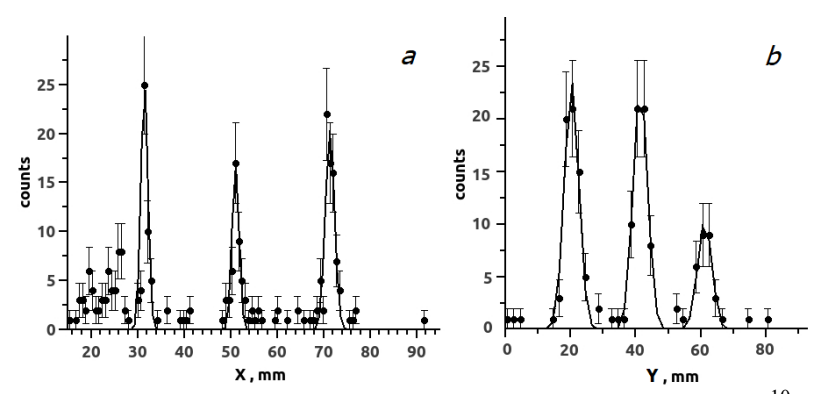

Fig. 2. Neutron coordinate distribution measured in the ${ }^{10} \mathrm{~B}$ detector at $\mathrm{U}=700 \mathrm{~V}$ with Cd-mask along: (a) X-axis; (b) Yaxis.

\section{Experiments}

\subsection{Thermal neutrons}

An important task is to measure the thermal neutron flux from the output channel of the moderator at various distances from its center. The ${ }^{10} \mathrm{~B}$ - and ${ }^{3} \mathrm{He}$-detectors was located along an axis of the channel. The ${ }^{3} \mathrm{He}$-detector was moved along $\mathrm{X}$-axis perpendicularly to the channel axis and it was located behind the ${ }^{10} \mathrm{~B}$-detector. A profile of the flux of the neutron source was measured by using both detectors. They were located at several distances from the moderator center. For example, they were placed at a distance of $204 \mathrm{~cm}$ from the center. The measured distribution of the neutron flux by ${ }^{10} \mathrm{~B}$-detector is shown in Fig.3(a). It was compared with the one which was measured with using of the moving ${ }^{3} \mathrm{He}$ detector (see Fig.3(b)). It can be note in the Fig.3(b) that the neutron flux is accompanied by a large background of scattered neutrons. It can be explained by a fact that the ${ }^{3} \mathrm{He}$ tube detector is sensitive to neutrons from all directions. At the same time, the ${ }^{10} \mathrm{~B}$-detector has a much lower sensitivity to the background of scattered neutrons.
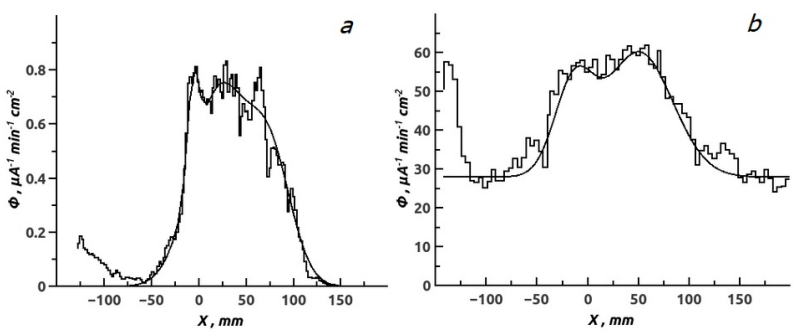

Fig. 3. Spatial neutron distribution from the moderator channel along the $\mathrm{X}$-axis which was measured by: (a) ${ }^{10} \mathrm{~B}$-detector at $\mathrm{U}=700 \mathrm{~V}$; (b) the moving ${ }^{3} \mathrm{He}$ tube counter at $\mathrm{U}=1400 \mathrm{~V}$.

The ${ }^{10} \mathrm{~B}$-detector gives a possibility to measure a time-of-flight. Time-of-flight measurements were performed with ${ }^{10} \mathrm{~B}$-detector and ${ }^{3} \mathrm{He}$ tube counter. The time-of-flight base for the ${ }^{3} \mathrm{He}$ tube counter was $\sim 7 \mathrm{~m}$, for the ${ }^{10} \mathrm{~B}$-detector $-3 \mathrm{~m}$. Therefore the time-of-flight scale must be substituted by the corresponding energy one. The neutron energy spectra obtained by the time-offlight technique with the ${ }^{10} \mathrm{~B}$-detector and ${ }^{3} \mathrm{He}$ tube counter are shown in Fig.4. Discrepancy in the shape of spectra can be explained by difference in the material and thickness of entrance windows. 
The accuracy and energy range of the time-of-flight measurement depends on the distance. The need for a large distance contradicts the requirement of a large solid angle. Therefore, as a rule, the time-of-flight measurement is precise enough only for thermal or epithermal neutrons.
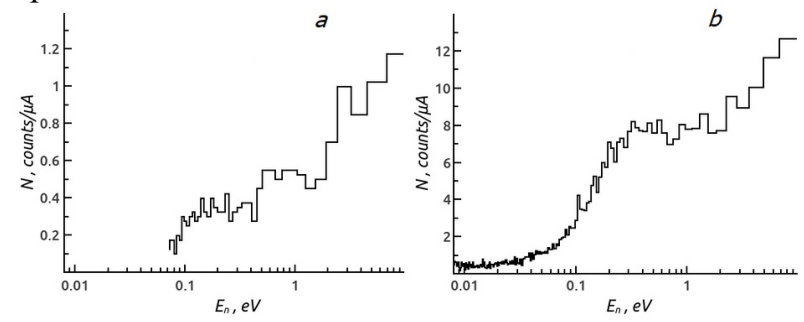

Fig. 4. Neutron energy spectra obtained by the time-of-flight technique. They were measured by: (a) ${ }^{10} \mathrm{~B}$-detector; (b) ${ }^{3} \mathrm{He}-$ tube counter.

\subsection{Simulations for thermal and fast neutrons}

Simulations gave that the position of maximum in the ionization loss spectrum corresponds to approximately $0.43 \mathrm{MeV}$ for the thermal neutron as it is shown in Fig.5(a). This made it possible to calibrate spectra on an energy scale. Simulations of the ionization loss spectrum for the $3 \mathrm{MeV}$ energy are presented in Fig.5(b).
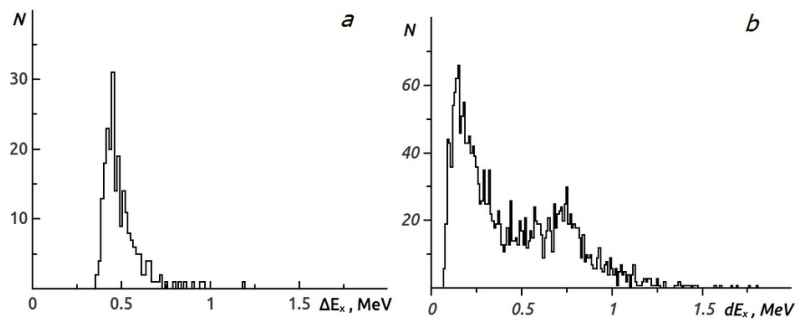

Fig. 5. Simulations of ionisation loss spectra with ${ }^{10} \mathrm{~B}$ detector in the first gas gap for: (a) thermal energy; (b) $3 \mathrm{MeV}$ energy.

An average ionization loss of the ${ }^{4} \mathrm{He}$ nucleus decreases with a increasing energy. An average ionization loss of the ${ }^{7} \mathrm{Li}$ nucleus increases with a increasing energy in our case. Note that, for a comparison with experiment the energy threshold must be taken into account.

\subsection{Fast neutrons}

An ionization loss signal from a thermal neutron is mainly caused by the emitted ${ }^{4} \mathrm{He}$ nucleus because ${ }^{7} \mathrm{Li}$ nuclei cannot penetrate into the gas gap. But if a neutron has an energy of several $\mathrm{MeV}$ the shape of pulse height spectra changes crucially. The ${ }^{7} \mathrm{Li}$ nuclei becomes capable to penetrate into the sensitive gas gaps [19].

To perform fast neutron experiments the moderator has been removed. The ${ }^{10} \mathrm{~B}$-detector was located at a distance of $310 \mathrm{~cm}$ from the center of the beryllium target at an angle of 43 with respect to the beam. The experimental ionization loss spectra for two gaps of detector are shown in Fig.6.

Neutrons with energies of several $\mathrm{MeV}$ transfer an additional energy to the emitted ${ }^{4} \mathrm{He}$ or ${ }^{7} \mathrm{Li}$ nuclei. The ${ }^{4} \mathrm{He}$ nucleus leaves less energy in the gas gap and causes the left peak in the spectrum. The ${ }^{7} \mathrm{Li}$ nucleus thus obtains enough energy to penetrate to the gap and leave a lot of energy in it [17].
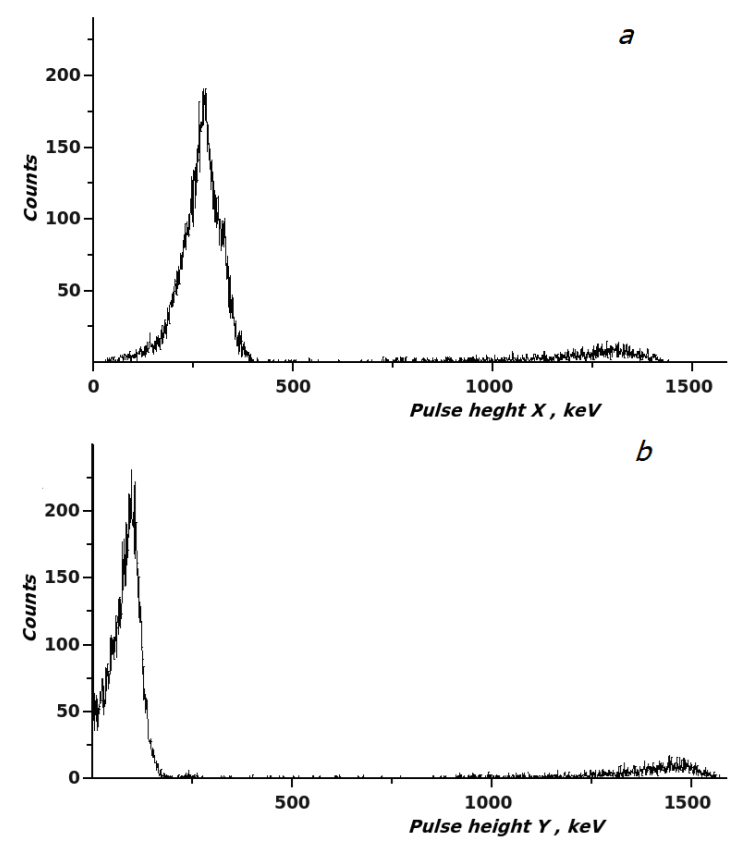

Fig. 6. The experimental ionisation loss spectra for the fast neutrons of several $\mathrm{MeV}$ with ${ }^{10} \mathrm{~B}$ detector for: (a) the 1-st gap; (b) the 2-nd gap.

Photoneutrons are produced on beryllium target. The yield of neutrons measured in the ${ }^{0} \mathrm{~B}$-detector depends on the electron beam energy as it is shown in Fig.7.

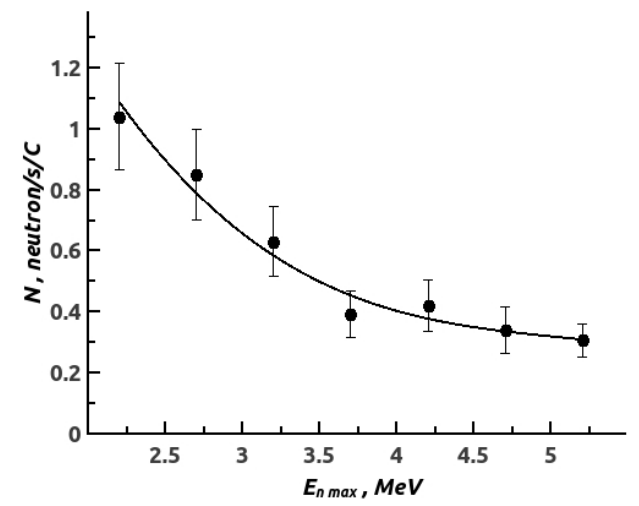

Fig. 7. The neutron counting rate in the ${ }^{10} \mathrm{~B}$-detector per sec per coulomb of the accelerator beam charge versus maximum energy of neutrons.

The number of detected neutrons per second per coulomb of the accelerator beam charge reduces with the maximum neutron energy.

The moderator was installed again for further experiments. But the moderator of the neutron source was configured to the flux which was enriched by fast neutrons of several $\mathrm{MeV}$ energy. The beryllium target was placed in the moderator center and channel was opened. The collimator of $3 \mathrm{~cm}$ in diameter was installed in the channel. The ${ }^{10} \mathrm{~B}$ detector was located at a distance of $119 \mathrm{~cm}$ from the target center at the output from the moderator channel. In order to remove slow neutrons from the flux, a $5 \mathrm{~mm}$ thick cadmium shield was placed 
in front of the detector. Two-dimension spatial distribution of neutrons with energies above the cadmium boundary $(>0.55 \mathrm{eV})$ and the neutron maximum energy $7 \mathrm{MeV}$ is shown in the Fig.8.

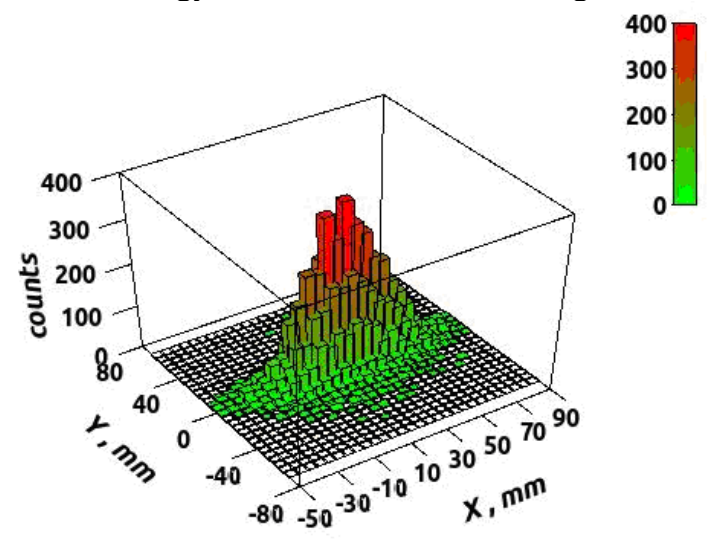

Fig. 8. The neutron two-dimension spatial distribution with the energy above $0.55 \mathrm{eV}$ and the maximum energy $7 \mathrm{MeV}$.

At the same time, the ${ }^{3} \mathrm{He}$-detector which was placed behind ${ }^{10} \mathrm{~B}$-detector detected only rare events. The efficiency of the ${ }^{3} \mathrm{He}$-detector to fast neutrons is extremely low because of the fast neutron cross section on ${ }^{3} \mathrm{He}$ nucleus is very small.

Ionization loss spectra which is produced in the first gap are shown in the Fig.9. The maximum neutron energy $E_{\mathrm{n} \max }$ was from 3 to $7 \mathrm{MeV}$.

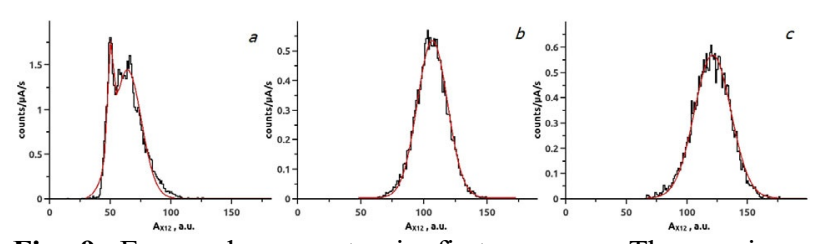

Fig. 9. Energy loss spectra in first gas gap. The maximum neutron energy $E_{\mathrm{n} \max }$ was: (a) $3 \mathrm{MeV}$; (b) $5 \mathrm{MeV}$; (c) $7 \mathrm{MeV}$.

We have found that the ionization loss and, hence, energy loss in the ${ }^{10} \mathrm{~B}$ detector gaps depends on the maximum neutron energy $E_{\mathrm{n} \max }$. The position of the maximum $P_{\max }$ in the pulse height spectrum varies monotonically versus the maximum neutron energy $E_{\text {n max }}$. This is demonstrated in Fig. 10.

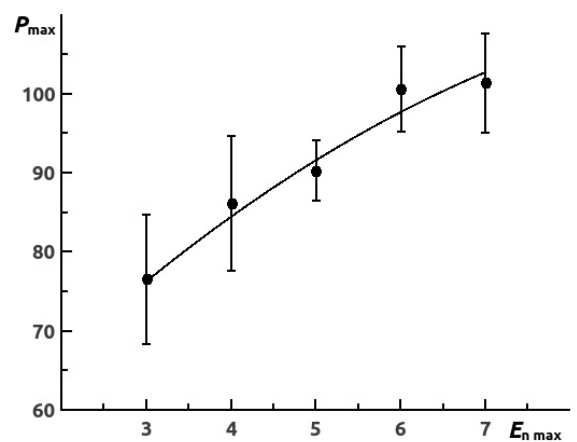

Fig. 10. The position of the maximum $P_{\text {max }}$ in the pulse height spectrum versus the maximum neutron energy $E_{\mathrm{n} \max }$.

The one layer detector has a $\sim 3 \cdot 10^{-2}$ efficiency for thermal neutrons and $\sim 10^{6}$ efficiency for several $\mathrm{MeV}$ neutrons. The efficiency could be enhanced by using many ${ }^{10} \mathrm{~B}$ layers. The detector will weakly distort the measured neutron field and it will have a negligible sensitivity to $\gamma$-quants.

\section{Conclusions}

The developed novel hybrid ${ }^{10} \mathrm{~B}$ detector demonstrates the possibility of separating thermal and fast neutrons. Experimentally measured pulse heights depend on the maximum neutron energy in the MeV-range. This allows to control the neutron flux energy. The obtained spatial resolutions for thermal neutrons along the $\mathrm{X}$ - and $\mathrm{Y}$ - axis were $\sim 2 \mathrm{~mm}$ and $4 \mathrm{~mm}$ respectively.

The detector has a very low sensitivity $\left(<10^{7}\right)$ to other types of radiation including $\gamma$-rays and weakly distorts neutron field due to small scattering and absorption in gas, aluminum and silicium of which such detector is built. The hybrid ${ }^{10} \mathrm{~B}$-detector is suited to study rare nuclear reactions.

We are grateful to L.V. Kravchuk for the interest to this work, and to S.V. Sabinin and V.V. Tishin for the technical support.

\section{References}

1. J. M. Tranquada, Guangyong Xu, I.A. Zaliznyak, J. Magn. Magn. Mater. 350, 148 (2014)

2. E. Kartini, M. Manavan, M.F. Collins, M. Avdeev, Physica B, Condens. Matter. Phys. 551, 320 (2018)

3. M.M. Castellanos, A. McAuley, J.E. Curtis, Comput. Struct. Biotech. J. 15, 117 (2017)

4. V.L. Aksenov, Physics-Uspekhi 45, 645 (2002)

5. S.I. Potashev, V.P. Zavarzina, A.A. Afonin, Bull. Lebedev Phys. Inst. 44, 271 (2017)

6. M.V. Avdeev, M. Balasoiu, V.L. Aksenov, V.M. Garamus, J. Kohlbrecher, D. Bica, L. Vekas, J. Magn. Magn. Mater. 270, 371 (2004)

7. D.A. Jacques, J. Trewhella, Protein Science 19, 642 (2010)

8. J. Fried, J.A. Harder, G.J. Mahler, D. Makowiecki, Nucl. Instrum. Methods in Phys. Res. Sect. A 478, 415 (2002)

9. T. Nakamura, K. Toh, N. Tsutsui, M. Ebine, A. Birumachi, K. Sakasai, J. Instrum. 12, C12025 (2017)

10. M. Katagiri, T. Nakamura, M. Ebine, A. Birumachi, Nucl. Instrum. Methods in Phys. Res. Sect. A 573, 149 (2007)

11. M.G. Strauss, R. Brenner, F.J. Lynch, C.B. Morgan, IEEE Trans. Nucl. Sci. 28, 800 (1981)

12. R. Beyer, E. Grossea, K. Heidel, J. Hutsch, A.R. Junghans, J. Klug, D. Legrady, R. Nolte, S. Rottger, M. Sobiella, A. Wagner, Nucl. Instrum. Methods in Phys. Res. Sect. A 575, 449 (2007)

13. M. Kohli, F. Allmendinger, W. Haubler, T. Schroder, M. Klein, M. Meven, U. Shmidt, Nucl. Instrum. Methods in Phys. Res. Sect. A 828, 242 (2016)

14. S. Uno, T. Uchida, M. Sekimoto, T. Murakami, K. Miyama, M. Shoji, E. Nakano, T. Koike, K. Morita, H. Satoh, T.Kamiyama, Y. Kiyanagi, Physics Procedia 26, 142 (2012) 
15. S. Potashev, Yu. Burmistrov, A. Drachev, S. Karaevsky, E. Konobeevski, S. Zuyev, J. Phys.: Conf. Ser. 798, 012160 (2017)

16. S.I. Potashev, Yu.M. Burmistrov, A.I. Drachev, S.Kh. Karaevsky, G.V. Solodukhov, J. Surf. Invest.: Xray, Synchrotron Neutron Tech., 12, 627 (2018)

17. S. Potashev, Yu. Burmistrov, A. Drachev, S. Karaevsky, E. Konobeevski, A. Kasparov, I. Meshkov, S. Zuyev, A. Afonin, V. Marin, V. Ponomarev, G. Solodukhov, KnE Energy \& Physics 12, 627 (2018)

18. S. Karaevsky, S. Potashev, A. Drachev, Yu. Burmistrov, Bull. Russ. Acad. Sci.: Phys. 82, 748 (2018)

19. R.A. Boie, J. Fischer, Y. Inagaki, F.C. Merritt, H.

Okuno, V. Radeka, Nucl. Instrum. Methods in Phys. Res. Sect. A 200, 533 (1982) 